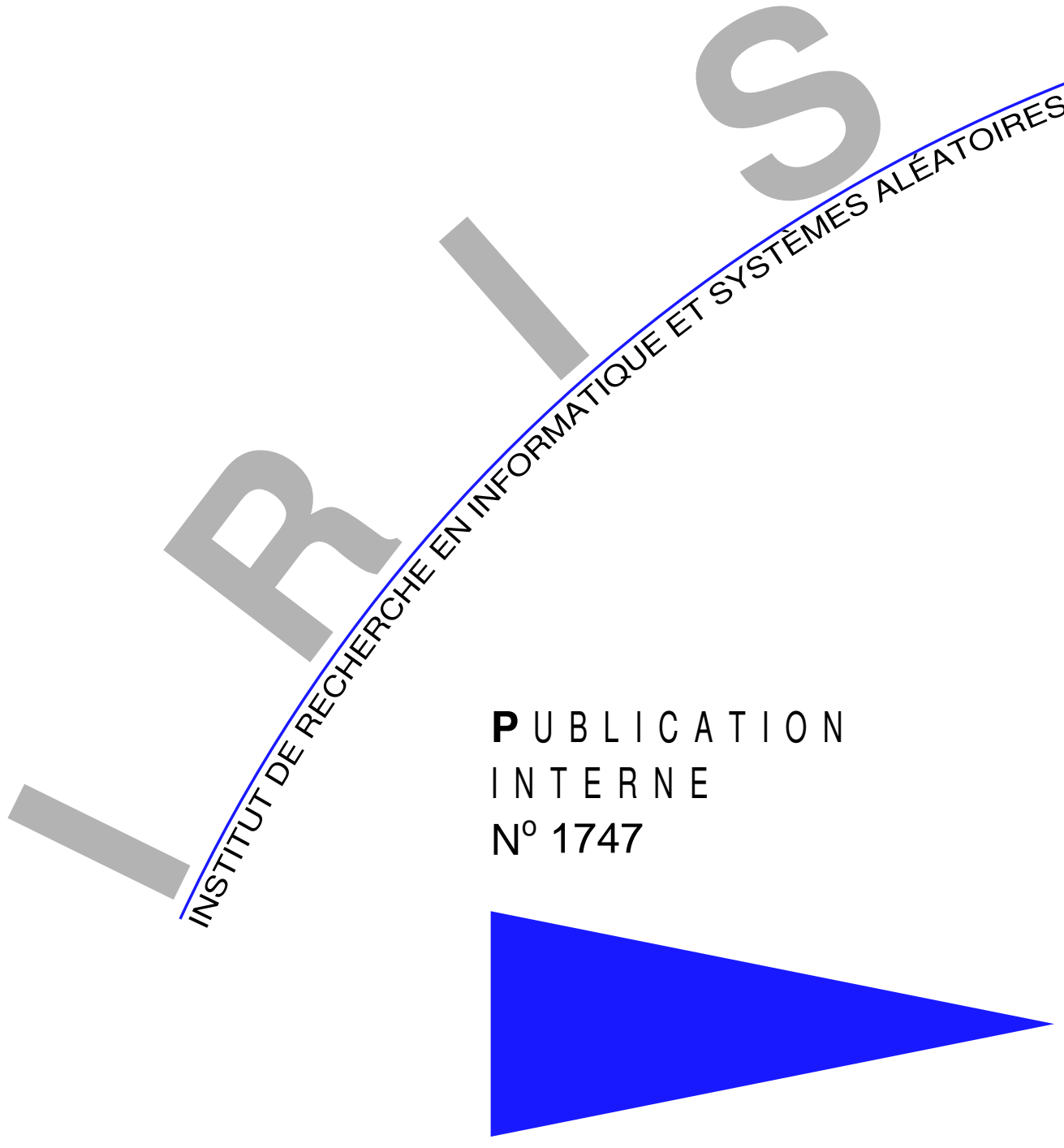

\title{
ADAPTIVE MULTILEVEL SPLITTING FOR RARE EVENT ANALYSIS
}

FRÉDÉRIC CÉROU , ARNAUD GUYADER 



\title{
Adaptive multilevel splitting for rare event analysis
}

\author{
Frédéric Cérou, Arnaud Guyader \\ Systèmes numériques \\ Projets Aspi \\ Publication interne $\mathrm{n}^{\circ} 1747$ - October 2005 - 27 pages
}

\begin{abstract}
The estimation of rare event probability is a crucial issue in areas such as reliability, telecommunications, aircraft management. In complex systems, analytical study is out of question and one has to use Monte Carlo methods. When rare is really rare, which means a probability less than $10^{-9}$, naive Monte Carlo becomes unreasonable. A widespread technique consists in multilevel splitting, but this method requires enough knowledge about the system to decide where to put the levels at hand. This is unfortunately not always possible. In this paper, we propose an adaptive algorithm to cope with this problem: the estimation is asymptotically consistent, costs just a little bit more than classical multilevel splitting and has the same efficiency in terms of asymptotic variance. In the one dimensional case, we prove rigorously the a.s. convergence and the asymptotic normality of our estimator, with the same variance as with other algorithms that use fixed crossing levels. In our proofs we mainly use tools from the theory of empirical processes, which seems to be quite new in the field of rare events.
\end{abstract}

Key-words: rare events, adaptive multilevel simulation, asymptotic normality

(Résumé : tsvp)

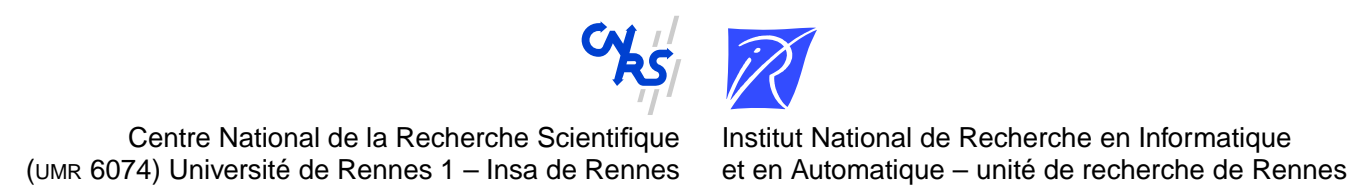




\section{Méthode multi-niveaux adaptative pour les événements rares}

Résumé : L'estimation de la probabilité d'un événement rare est un problème crucial dans des domaines tels que la fiabilité, les télécommunications, le contrôle aérien. Dans des systèmes complexes, l'étude analytique est hors de portée, et on doit utiliser une méthode de Monte Carlo. Lorsque l'événement est vraiment rare, disons ayant une probabilité plus petite que $10^{-9}$, une approche Monte Carlo nä̈ve ne marche pas. Une technique courante consiste à utiliser des niveaux de branchement, mais cette méthode nécessite une connaissance suffisante du système pour choisir où mettre les différents niveaux. Cela n'est malheureusement pas toujours possible. Dans cet article, nous proposons un nouvel algorithme adaptatif pour résoudre ce problème : l'estimateur est asymptotiquement consistant, est juste un peu plus coûteux que la méthode multi-niveaux classique, et a la même efficacité en terme de variance asymptotique. Dans le cas unidimensionnel, nous montrons rigoureusement la convergence presque sûre et la normalité asyptotique de notre estimateur, avec la même variance que les autres algorithmes utilisant des niveaux fixés. Les preuves utilisent des outils issus des processus empiriques, une approche qui semble nouvelle dans le champ des événements rares.

Mots clés : événements rares, simulation multi-niveau adaptative, normalité asymptotique 


\section{Introduction}

Let $\left(X_{t}\right)_{t \geq 0}$ be a strong Markov process with values in $\mathbb{R}$. Suppose that $X_{0}=x_{0}>0$, the origin 0 is an attractive point and $M \gg 0$ is a barrier that the process is very unlikely to reach. We would like to estimate the probability that $\left(X_{t}\right)$ reaches $M$ before coming back to 0 : this is our rare event.

For the sake of simplicity, we suppose that the trajectories are continuous. The point 0 is "attractive" means that if we define

$$
T_{0}=\inf \left\{t \geq 0: X_{t}=0\right\},
$$

the stopping time $T_{0}$ is such that : $\mathbb{E}\left[T_{0}\right]<+\infty$.

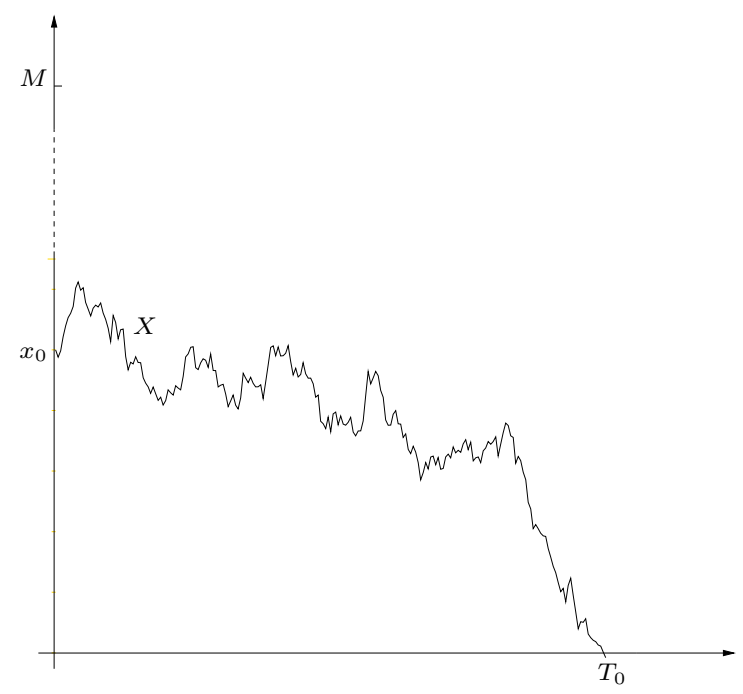

Figure 1: An example of trajectory for the Markov process.

Since $\left(X_{t}\right)$ tends to decrease to 0 , it is clear that if we denote

$$
T_{M}=\inf \left\{t \geq 0: X_{t}=M\right\},
$$

we have

$$
\mathbb{P}\left(T_{M}<T_{0}\right) \approx 0 .
$$

PI ${ }^{\circ} 1747$ 
Let us consider the situation when this very small probability $\mathbb{P}\left(T_{M}<T_{0}\right)=\alpha$ is strictly positive. We want to get an estimation $\hat{\alpha}$ of $\alpha$.

When the process $\left(X_{t}\right)_{t \geq 0}$ is not simple, the usual way to cope with this kind of problem is to use Monte Carlo techniques. The most natural one is to simulate $n$ i.i.d. trajectories of the process, to count those who reach $M$ before 0 , and to compute the ratio. Unfortunately, when the event is really rare, this is completely unrealistic. Another idea, called Importance Sampling, is to simulate trajectories with respect to another reference probability, so that the rare event becomes less rare, and then to correct the estimation via the importance function (which corresponds to a Radon-Nikodym derivative). But it is a difficult problem to find another suitable reference probability, especially when the system in study is very complex.

In such a situation, a classical method is the one of multilevel splitting : the idea dates back to 1951 with the work of Kahn and Harris in the setting of particle transmission [12]. Roughly speaking, the principle is to multiply the trajectories that approach the event of interest and to let the other die. It is much more simple to implement than Importance Sampling but it requires some a priori knowledge on the system also, precisely : when and how many times shall one split the trajectories? Hereafter we propose a new method, called adaptive multilevel splitting, which uses the splitting idea, but where the splitting levels are determined during the simulations.

The article is organized as follows. Section 2 describes the algorithm and the estimator of the rare event probability. Section 3 proves the consistency of the estimator. Section 4 establishes the asymptotic normality, with a very simple expression of the variance. Section 5 illustrates these results on a toy example. Finally, section 6 compares this adaptive algorithm with existing versions of multilevel splitting and shows how the method can be used in various situations.

\section{The algorithm}

The structure of the algorithm is the following : to approach the barrier $M$ of interest, we proceed in several steps. Except the first and the last ones, all steps are equivalent. In what follows, $n$ denotes the number of particles which are simulated, and $k$ denotes the number of particles that we do not throw away from one step to another.

- Step 1 : simulate $n$ i.i.d. trajectories $\left(X_{t}^{j}\right)_{t \geq 0}$ according to the law of the process $\left(X_{t}\right)_{t \geq 0}$ and with common initial condition

$$
\forall j \in\{1, \ldots, n\} \quad X_{0}^{j}=x_{0}>0 .
$$




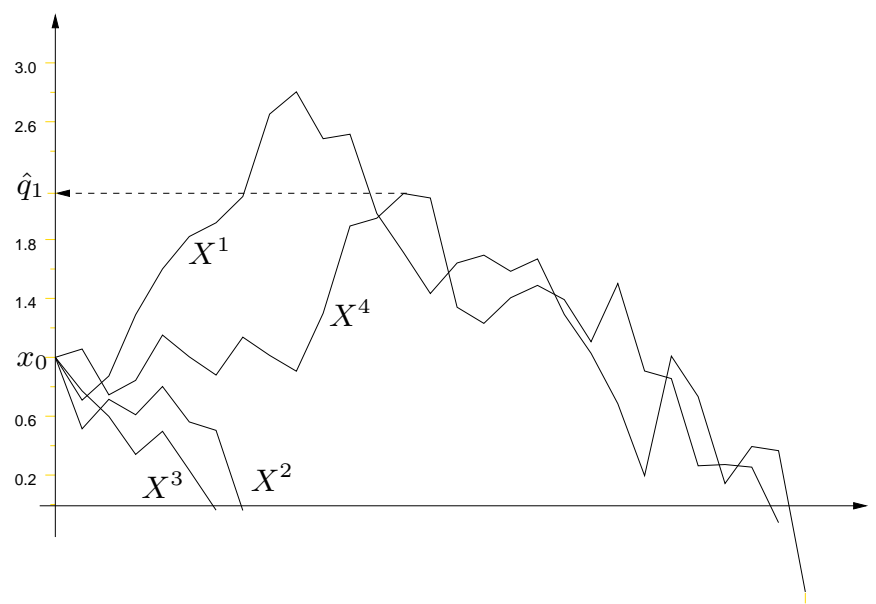

Figure 2: The first step of the algorithm, with $n=4$ and $k=1$.

Wait until all trajectories have reached $0:$ for the $j$-th particle, this requires time $T_{0}^{j}$, with $\mathbb{E}\left[T_{0}^{j}\right]=\mathbb{E}_{x_{0}}\left[T_{0}\right]<+\infty$. Denote

$$
S_{n, j}^{1}=\sup _{0 \leq t \leq T_{0}^{j}} X_{t}^{j}
$$

and sort the sample $\left(S_{n, 1}^{1}, \ldots, S_{n, n}^{1}\right)$ in increasing order :

$$
S_{n,(1)}^{1} \leq \cdots \leq S_{n,(n-k)}^{1} \leq \cdots \leq S_{n,(n)}^{1} .
$$

Keep in memory the quantity (see Figure 2)

$$
\hat{q}_{1}=S_{n,(n-k)}^{1} .
$$

- Step 2 : Keep $\left(S_{n,(n-k+1)}^{1}, \ldots, S_{n,(n)}^{1}\right)$ unchanged, but denote them simply $\left(S_{n, n-k+1}^{2}, \ldots, S_{n, n}^{2}\right)$. Simulate $(n-k)$ trajectories $\left(X_{t}^{j}\right)_{t \geq 0}$ from initial point $\hat{q}_{1}$. Wait until all these $(n-k)$ trajectories have reached $0:$ for the $j$-th particle, this requires time $T_{0}^{j}$, with $\mathbb{E}\left[T_{0}^{j}\right]=\mathbb{E}_{\hat{q}_{1}}\left[T_{0}\right]<+\infty$. For each $j \in\{1, \ldots, n-k\}$, denote

$$
S_{n, j}^{2}=\sup _{0 \leq t \leq T_{0}^{j}} X_{t}^{j}
$$


and sort the sample $\left(S_{n, 1}^{2}, \ldots, S_{n, n-k}^{2}, S_{n, n-k+1}^{2}, \ldots, S_{n, n}^{2}\right)$ in increasing order :

$$
S_{n,(1)}^{2} \leq \cdots \leq S_{n,(n-k)}^{2} \leq \cdots \leq S_{n,(n)}^{2} .
$$

Keep in memory the quantity (see Figure 3)

$$
\hat{q}_{2}=S_{n,(n-k)}^{2}
$$

- ...

- Repeat the procedure until $\hat{q}_{N+1} \geq M$. Among the sample $\left(S_{n, 1}^{N}, \ldots, S_{n, n}^{N}\right)$, there is a proportion $r>0$ of them that are actually bigger than $M$.

- Compute the probability estimate. If we denote $p=\frac{k}{n}$, then the estimate of the rare event is simply

$$
\hat{\alpha}_{n}=r p^{N} .
$$

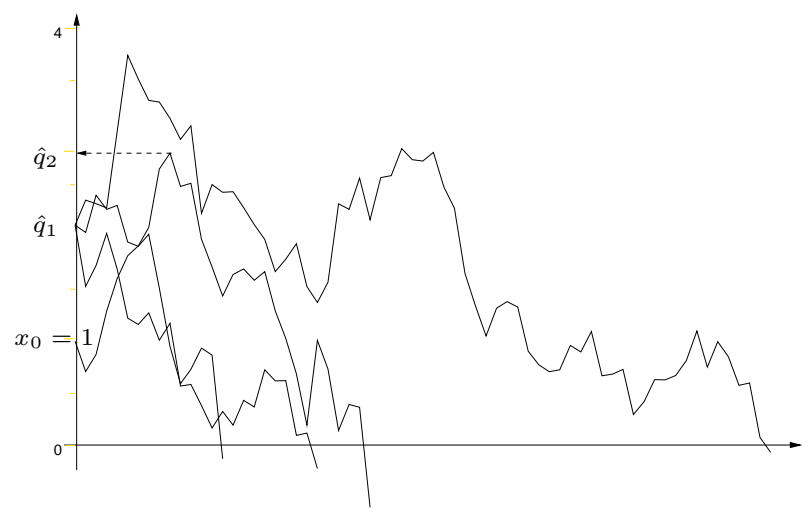

Figure 3: The second step of the algorithm, with $n=4$ and $k=1$.

\section{Consistency}

At the end of step $l$, it is clear that the random variables $\left(S_{n, j}^{l}\right)_{1 \leq j \leq n}$ are i.i.d. according to the following law :

$$
S_{n, j}^{l} \sim \mathcal{D}\left(\sup _{0 \leq t \leq T_{0}} X_{t}^{x_{0}} \mid \sup _{0 \leq t \leq T_{0}} X_{t}^{x_{0}} \geq \hat{q}_{l-1}\right)
$$

with the convention that $\hat{q}_{0}=x_{0}$. Thanks to the strong Markov property of the process and the continuity of its trajectories, we can write it a little bit simpler :

$$
S_{n, j}^{l} \sim \mathcal{D}\left(\sup _{0 \leq t \leq T_{0}} X_{t}^{\hat{q}_{l-1}} \mid \hat{q}_{l-1}\right) .
$$


Let us also denote $S=\sup _{0 \leq t \leq T_{0}} X_{t}^{x_{0}}$, and $F$ its distribution function. We suppose that $F$ is continuous (note that this property is not implied by the continuity of trajectories). We now define $\mathbf{F}$ a deterministic function of two variables $\left(q_{1}, q_{2}\right)$ as follows:

$$
\mathbf{F}\left(q_{1}, q_{2}\right)=\mathbb{P}\left(S \leq q_{2} \mid S>q_{1}\right) .
$$

Since $F$ is continuous, we have the obvious identity

$$
\mathbf{F}\left(q_{1}, q_{2}\right)=\frac{F\left(q_{2}\right)-F\left(q_{1}\right)}{1-F\left(q_{1}\right)} .
$$

Thus each $S_{n, j}^{l}$ has the distribution function $\mathbf{F}\left(\hat{q}_{l-1},.\right)$. Note that at each step, the algorithm is estimating $\mathbf{F}\left(\hat{q}_{l-1}, \hat{q}_{l}\right)$ by $q=(1-p)$. Since $F$ is continuous, the random variables $U_{n, 1}^{l}, \ldots, U_{n, n}^{l}$, with

$$
U_{n, j}^{l}=\mathbf{F}\left(\hat{q}_{l-1}, S_{n, j}^{l}\right),
$$

are identically distributed with uniform law on $[0,1]$ and row-wise independent. In fact, the proof of consistency mainly relies on this simple argument. Before stating the theorem, we sum up the assumptions on our model.

Hypothesis $(\mathcal{H})$. The strongly Markov process $\left(X_{t}\right)_{t \geq 0}$ starts from $x_{0}>0$, with 0 as an attractive point. $\left(X_{t}\right)_{t>0}$ has time-continuous trajectories and the distribution function $F$ of the random variable $S=\sup _{0 \leq t \leq T_{0}} X_{t}^{x_{0}}$ is continuous.

Theorem 1. Under assumption $(\mathcal{H})$, we have

$$
\hat{\alpha}_{n} \underset{n \rightarrow \infty}{\stackrel{a . s .}{\longrightarrow}} \alpha
$$

In order to prove this theorem, will need two simple lemmas.

Lemma 1. Let $\left(U_{n, j}\right)_{1 \leq j \leq n}$ be a triangular array of identically distributed random variables with uniform law on $[0,1]$ and row-wise independent. Let $k_{n}=\lfloor n p\rfloor$, then

$$
U_{n,\left(n-k_{n}\right)}^{\stackrel{a . s .}{\longrightarrow}} q=1-p .
$$

Proof. Let us denote by $\mathbb{G}_{n}$ the empirical distribution function of $\left(U_{n, j}\right)_{1 \leq j \leq n}$, and $G$ the distribution function of the uniform law on $[0,1]$, that is the identity on $[0,1]$. First note the basic identity $\left\|\mathbb{G}_{n}-G\right\|_{\infty}=\left\|\mathbb{G}_{n}^{-1}-G\right\|_{\infty}($ see $[15]$ page 86$)$, and $U_{n,\left(n-k_{n}\right)}=\mathbb{G}_{n}^{-1}\left(1-\frac{k_{n}}{n}\right)$. We have also

$$
\left|U_{n,\left(n-k_{n}\right)}-q\right| \leq\left\|\mathbb{G}_{n}^{-1}-G\right\|_{\infty}+\left|p-k_{n} / n\right| .
$$

Using Dvoretsky-Kiefer-Wolfowitz (DKW) inequality (see [14, 16]) we have

$$
\mathbb{P}\left(\left\|\mathbb{G}_{n}-G\right\|_{\infty}>\frac{1}{\log n}-\left|q-k_{n} / n\right|\right) \leq 2 \exp \left[-2 n\left(\frac{1}{\log n}-\left|p-k_{n} / n\right|\right)^{2}\right],
$$


which implies that

$$
\mathbb{P}\left(\left|U_{n,\left(n-k_{n}\right)}-q\right|>\frac{1}{\log n}\right) \leq 2 \exp \left[-2 n\left(\frac{1}{\log n}-\left|p-k_{n} / n\right|\right)^{2}\right] .
$$

We conclude using Borel-Cantelli lemma and the convergence of the series on the right hand side, having noted that $\left|p-k_{n} / n\right| \leq \frac{1}{n}$.

Lemma 2. Let $\left(V_{n, j}\right)_{1 \leq j \leq n}$ be a triangular array of random variables row-wise independent and identically distributed. Let $H_{n}$ be the distribution function of the $n^{\text {th }}$ row, and $\mathbb{H}_{n}$ its empirical distribution function. Then

$$
\left\|H_{n}-\mathbb{H}_{n}\right\|_{\infty} \underset{n \rightarrow \infty}{\stackrel{a . s .}{\longrightarrow}} 0 .
$$

Proof. This is again a simple application of DKW inequality, since

$$
\mathbb{P}\left(\left\|\mathbb{H}_{n}-H_{n}\right\|_{\infty}>\frac{1}{\log n}\right) \leq 2 \exp \left[-2 n\left(\frac{1}{\log n}\right)^{2}\right] .
$$

We conclude using Borel-Cantelli lemma and the convergence of the series on the right hand side.

Proof of the theorem. First of all, we shall see that for all $l$ :

$$
\mathbf{F}\left(\hat{q}_{l-1}, \hat{q}_{l}\right) \underset{n \rightarrow \infty}{\stackrel{a . s .}{\longrightarrow}} 1-p .
$$

This property is a direct application of the lemma 1.

Then, we should notice that

$$
\prod_{k=1}^{l}\left(1-\mathbf{F}\left(\hat{q}_{k-1}, \hat{q}_{k}\right)\right)=\prod_{k=1}^{l} \frac{1-F\left(\hat{q}_{k}\right)}{1-F\left(\hat{q}_{k-1}\right)}=1-F\left(\hat{q}_{l}\right)=\mathbb{P}\left(\sup _{0 \leq t \leq T_{0}} X_{t}^{x_{0}}>\hat{q}_{l} \mid \hat{q}_{l}\right) .
$$

Next we will show that for all $l$

$$
\left|\prod_{k=1}^{l}\left(1-\mathbf{F}\left(\hat{q}_{k-1}, \hat{q}_{k}\right)\right)-p^{l}\right| \underset{n \rightarrow \infty}{\stackrel{a . s .}{\longrightarrow}} 0 .
$$

We already have the convergence for $l=1$. Then we proceed by induction. Assume that the previous convergence is true for some $l \geq 1$. Then we use that the product of two random variables will a.s. converge to the product of the limits.

Now we focus on the last step. Assume first that $\frac{\log \alpha}{\log p}$ is not an integer. Let $N=\left\lfloor\frac{\log \alpha}{\log p}\right\rfloor$. Then we have that a.s. for $n$ large enough,

$$
\prod_{k=1}^{N+1}\left[1-\mathbf{F}\left(\hat{q}_{k-1}, \hat{q}_{k}\right)\right]<\alpha<\prod_{k=1}^{N}\left[1-\mathbf{F}\left(\hat{q}_{k-1}, \hat{q}_{k}\right)\right]
$$


that is

$$
1-F\left(\hat{q}_{N+1}\right)<\alpha<1-F\left(\hat{q}_{N}\right)
$$

which implies that

$$
\hat{q}_{N}<M<\hat{q}_{N+1},
$$

so that, a.s. for $n$ large enough, the algorithm stops after $N$ iterations. Let us denote by $\mathbb{F}_{n, N}$ the empirical distribution function of the $\left\{S_{n, j}^{N}, 1 \leq j \leq n\right\}$. Using lemma 2 , we have that a.s.

$$
\left|\mathbf{F}\left(\hat{q}_{N}, M\right)-\mathbb{F}_{n, N}(M)\right| \leq\left\|\mathbf{F}\left(\hat{q}_{N}, .\right)-\mathbb{F}_{n, N}\right\|_{\infty} \underset{n \rightarrow \infty}{\stackrel{a . s .}{\longrightarrow}} 0 .
$$

On the other hand, using the definition of $N$, we have that

$$
\prod_{k=1}^{N}\left[1-\mathbf{F}\left(\hat{q}_{k-1}, \hat{q}_{k}\right)\right] \cdot\left[1-\mathbf{F}\left(\hat{q}_{N}, M\right)\right]=1-F(M)=\alpha,
$$

which implies:

$$
\lim _{n \rightarrow+\infty}\left[1-\mathbb{F}_{n, N}(M)\right]=\frac{\alpha}{p^{N}} \text { a.s. }
$$

So we get

$$
\lim _{n \rightarrow+\infty} \hat{\alpha}_{n}=\lim _{n \rightarrow+\infty} p^{N}\left[1-\mathbb{F}_{n, N}(M)\right]=p^{N} \frac{\alpha}{p^{N}}=\alpha,
$$

which gives the estimate consistency.

Finally, we consider the case when $\frac{\log \alpha}{\log p}$ is an integer. Again we set $N=\frac{\log \alpha}{\log p}$. In this case, using the same arguments, we have

$$
\prod_{k=1}^{N}\left[1-\mathbf{F}\left(\hat{q}_{k-1}, \hat{q}_{k}\right)\right] \underset{n \rightarrow \infty}{\stackrel{a . s .}{\longrightarrow}} p^{N}=\alpha .
$$

So for $n$ large enough, the algorithm stops after $N$ or $(N+1)$ steps. We have to consider two cases: either $\hat{q}_{N} \geq M$, or $\hat{q}_{N}<M$, and the estimate may be written as

$$
\hat{\alpha}_{n}=p^{N-1}\left[1-\mathbb{F}_{n, N}(M)\right] \mathbb{1}_{\left\{\hat{q}_{N} \geq M\right\}}+p^{N}\left[1-\mathbb{F}_{n, N+1}(M)\right] \mathbb{1}_{\left\{\hat{q}_{N}<M\right\}} .
$$

We also have, using lemma 2 in the same way as we did in the first part of the proof

$$
\lim _{n \rightarrow+\infty}\left[1-\mathbb{F}_{n, N-1}(M)\right]=p
$$

PI ${ }^{\circ} 1747$ 
and

$$
\lim _{n \rightarrow+\infty}\left[1-\mathbb{F}_{n, N}(M)\right]=1
$$

Then we get

$$
\left|\hat{\alpha}_{n}-\alpha\right| \leq\left|p^{N-1}\left[1-\mathbb{F}_{n, N}(M)\right]-p^{N}\right| \cdot \mathbb{1}_{\left\{\hat{q}_{N} \geq M\right\}}+\left|p^{N}\left[1-\mathbb{F}_{n, N+1}(M)\right]-p^{N}\right| \cdot \mathbb{1}_{\left\{\hat{q}_{N}<M\right\}},
$$

where both terms tend a.s. to 0 , which concludes the proof.

Remark. If we suppose that $F^{-1}$ is continuous, the proof is shorter, since we do not need then lemmas 1 and 2. Indeed, in that case, classical results say that empirical quantiles converge almost surely towards the true quantiles (see for example [16], Lemma 21.2 p.305).

\section{Asymptotic normality}

Now we are interested in the variance of this estimation. Let us denote $\rho=\alpha p^{-N}$.

Theorem 2. Under the assumptions we have made so far, we have

$$
\sqrt{n}\left(\alpha-\hat{\alpha}_{n}\right) \underset{n \rightarrow+\infty}{\stackrel{\mathcal{D}}{\longrightarrow}} \mathcal{N}\left(0, \sigma^{2}\right),
$$

with

$$
\sigma^{2}=\alpha^{2}\left(N \frac{1-p}{p}+\frac{1-\rho}{\rho}\right) .
$$

In order to prove this theorem, we will need the following lemmas.

Lemma 3. Let $\left(U_{n, j}\right)_{1 \leq j \leq n}$ be a triangular array of identically distributed random variables with uniform law on $[0,1]$ and row-wise independent. Let $k_{n}=\lfloor n p\rfloor$, then

$$
\sqrt{n}\left(U_{n,\left(n-k_{n}\right)}-q\right) \underset{n \rightarrow \infty}{\stackrel{\mathcal{D}}{\longrightarrow}} \mathcal{N}(0, p(1-p)) .
$$

Proof. This is a direct application of theorem 8.5.1 page 223 of [1] to $\mathcal{U}(0,1)$-distributed i.i.d. random variables.

We recall now a very classical probabilistic result.

Lemma 4. Let $\left\{V_{n}, n \in \mathbb{N}\right\}$ and $\left\{W_{n}, n \in \mathbb{N}\right\}$ be two sequences of random variables such that

$$
V_{n} \underset{n \rightarrow \infty}{\stackrel{\mathcal{D}}{\longrightarrow}} V \text { and } W_{n} \underset{n \rightarrow \infty}{\stackrel{a . s .}{\longrightarrow}} 0
$$


Then

$$
V_{n}+W_{n} \underset{n \rightarrow \infty}{\stackrel{\mathcal{D}}{\longrightarrow}} V
$$

and

$$
V_{n} W_{n} \underset{n \rightarrow \infty}{\stackrel{a . s .}{\longrightarrow}} 0 .
$$

The following lemma, intuitively clear, is very useful in practice : roughly speaking, if we are only interested in the order statistics of the supremum, everything happens as if we had uniform and independent random variables.

Lemma 5. Let $l \geq 1, \hat{q}_{l}, \hat{q}_{l+1}, \mathbf{F}$ and $k_{n}$ be as before. For any test function $\varphi: \mathbb{R} \rightarrow \mathbb{R}$,

$$
\mathbb{E}\left[\varphi\left(\mathbf{F}\left(\hat{q}_{l}, \hat{q}_{l+1}\right)\right) \mid \hat{q}_{l}\right]=\mathbb{E}\left[\varphi\left(U_{\left(n-k_{n}\right)}\right)\right],
$$

with $\left(U_{n, j}\right)_{1 \leq j \leq n}$ a triangular array of identically distributed random variables with uniform law on $[0,1]$ and row-wise independent.

The last lemma is a little bit more technical.

Lemma 6. Let $\left(\rho_{n}\right)$ be a sequence of random variables and $\rho$ a deterministic constant such that

$$
\begin{gathered}
\forall n \in \mathbb{N}, \rho_{n} \in(0,1) \text { and } \rho \in(0,1), \\
\rho_{n} \underset{n \rightarrow \infty}{\stackrel{a . s .}{\longrightarrow}} \rho,
\end{gathered}
$$

and

$$
\sqrt{n}\left(\rho_{n}-\rho\right) \underset{n \rightarrow \infty}{\stackrel{\mathcal{D}}{\longrightarrow}} \mathcal{N}\left(0, \sigma^{2}\right)
$$

Let us consider next a triangular array $\left\{B_{i j}, i \leq j\right\}$ of random variables, with the $n^{\text {th }}$ row being conditionally to $\rho_{n}$ i.i.d. Bernoulli trials, of parameter $\rho_{n}$ (i.e. for all $i, n$, $\left.\mathbb{P}\left(B_{i n}=1 \mid \rho_{n}\right)=\rho_{n}=1-\mathbb{P}\left(B_{i n}=0 \mid \rho_{n}\right)\right)$. Then we have the following result:

$$
\sqrt{n}\left(\frac{1}{n} \sum_{j=1}^{n} B_{j n}-\rho\right) \underset{n \rightarrow \infty}{\stackrel{\mathcal{D}}{\longrightarrow}} \mathcal{N}\left(0, s^{2}\right)
$$

with $s^{2}=\sigma^{2}+\rho(1-\rho)$. 
Proof. In the sequel the notation $o($.$) refers to a.s. convergence. Let us first consider the$ conditional characteristic function

$$
\begin{aligned}
\phi_{n, \rho_{n}}(t) & =\mathbb{E}\left[\exp \left(i t \sqrt{n}\left(\frac{1}{n} \sum_{j=1}^{n} B_{j n}-\rho\right)\right) \mid \rho_{n}\right] \\
& =e^{-i t \rho \sqrt{n}}\left(\rho_{n} e^{\frac{i t}{\sqrt{n}}}+\left(1-\rho_{n}\right)\right)^{n} \\
& =\exp \left[-i t \rho \sqrt{n}+n \log \left(\rho_{n} e^{\frac{i t}{\sqrt{n}}}+\left(1-\rho_{n}\right)\right)\right] \\
& =\exp \left[-i t \rho \sqrt{n}+n \log \left(\rho_{n}\left(1+\frac{i t}{\sqrt{n}}-\frac{t^{2}}{2 n}+o\left(\frac{1}{n}\right)\right)+1-\rho_{n}\right)\right] \\
& =\exp \left[-i t \rho \sqrt{n}+n \log \left(1+\frac{i t \rho_{n}}{\sqrt{n}}-\frac{\rho_{n} t^{2}}{2 n}+o\left(\frac{1}{n}\right)\right)\right] .
\end{aligned}
$$

Then taking the expectation and developing the log,

$$
\begin{aligned}
\mathbb{E}\left[\phi_{n, \rho_{n}}(t)\right] & =\mathbb{E}\left[\exp \left[-i t \rho \sqrt{n}+n\left(\left(\frac{i t \rho_{n}}{\sqrt{n}}-\frac{\rho_{n} t^{2}}{2 n}\right)-\frac{1}{2}\left(\frac{i t \rho_{n}}{\sqrt{n}}-\frac{\rho_{n} t^{2}}{2 n}\right)^{2}+o\left(\frac{1}{n}\right)\right)\right]\right] \\
& =\mathbb{E}\left[\exp \left[i t \sqrt{n}\left(\rho_{n}-\rho\right)-\frac{1}{2} \rho_{n}\left(1-\rho_{n}\right) t^{2}+o(1)\right]\right]
\end{aligned}
$$

where

$$
\frac{1}{2} \rho_{n}\left(1-\rho_{n}\right) t^{2}+o(1) \underset{n \rightarrow \infty}{\stackrel{a . s .}{\longrightarrow}} \frac{1}{2} \rho(1-\rho),
$$

and

$$
\sqrt{n}\left(\rho_{n}-\rho\right) \underset{n \rightarrow \infty}{\stackrel{\mathcal{D}}{\longrightarrow}} \mathcal{N}\left(0, \sigma^{2}\right),
$$

from which we get the convergence of the pair and then

$$
\mathbb{E}\left[\phi_{n, \rho_{n}}(t)\right] \underset{n \rightarrow \infty}{\longrightarrow} \exp \left[-\frac{1}{2} \sigma^{2} t^{2}-\frac{1}{2} \rho(1-\rho) t^{2}\right] .
$$

The limit is the characteristic function of a random variable of law $\mathcal{N}\left(0, \sigma^{2}+\rho(1-\rho)\right)$, which concludes the proof of the lemma.

Now we are able to prove the theorem.

Proof of the theorem. We proceed like in the proof of theorem 1. We begin by seeing that for all $l$

$$
\sqrt{n}\left(1-\mathbf{F}\left(\hat{q}_{i}, \hat{q}_{i+1}\right)-p\right) \underset{n \rightarrow+\infty}{\stackrel{\mathcal{D}}{\longrightarrow}} \mathcal{N}(0, p(1-p)) .
$$


This property is a direct application of lemma 3 and lemma 5 . We will use the following identity :

$$
a b-c d=(a-c)(b-d)+(a-c) d+(b-d) c .
$$

Now we proceed by induction :

$$
\begin{aligned}
& \sqrt{n}\left(\prod_{k=1}^{l+1}\left[1-\mathbf{F}\left(\hat{q}_{k-1}, \hat{q}_{k}\right)\right]-p^{l+1}\right) \\
& =\quad \sqrt{n}\left(\prod_{k=1}^{l}\left[1-\mathbf{F}\left(\hat{q}_{k-1}, \hat{q}_{k}\right)\right]-p^{l}\right)\left(1-\mathbf{F}\left(\hat{q}_{l}, \hat{q}_{l+1}\right)-p\right) \\
& \quad+p \sqrt{n}\left(\prod_{k=1}^{l}\left[1-\mathbf{F}\left(\hat{q}_{k-1}, \hat{q}_{k}\right)\right]-p^{l}\right)+p^{l} \sqrt{n}\left(1-\mathbf{F}\left(\hat{q}_{l}, \hat{q}_{l+1}\right)-p\right) .
\end{aligned}
$$

For the first term, using equation (1), we know that :

$$
1-\mathbf{F}\left(\hat{q}_{l}, \hat{q}_{l+1}\right)-p \underset{n \rightarrow+\infty}{\stackrel{a . s .}{\longrightarrow}} 0
$$

and by induction hypothesis

$$
\sqrt{n}\left(\prod_{k=1}^{l}\left[1-\mathbf{F}\left(\hat{q}_{k-1}, \hat{q}_{k}\right)\right]-p^{l}\right) \underset{n \rightarrow+\infty}{\stackrel{\mathcal{D}}{\longrightarrow}} \mathcal{N}\left(0, \sigma_{l}^{2}\right) .
$$

So that, thanks to lemma 4 , we have

$$
\sqrt{n}\left(\prod_{k=1}^{l}\left[1-\mathbf{F}\left(\hat{q}_{k-1}, \hat{q}_{k}\right)\right]-p^{l}\right)\left(1-\mathbf{F}\left(\hat{q}_{l}, \hat{q}_{l+1}\right)-p\right) \underset{n \rightarrow+\infty}{\stackrel{a . s .}{\longrightarrow}} 0 .
$$

We want to prove that the other terms in equation (2) both converge in distribution. For this we use the characteristic function.

$$
\phi_{n}(t)=\mathbb{E}\left[\exp \left(i t\left(p \sqrt{n}\left(\prod_{k=1}^{l}\left[1-\mathbf{F}\left(\hat{q}_{k-1}, \hat{q}_{k}\right)\right]-p^{l}\right)+p^{l} \sqrt{n}\left(1-\mathbf{F}\left(\hat{q}_{l}, \hat{q}_{l+1}\right)-p\right)\right)\right)\right] .
$$

Thanks to the strong Markov property of the process :

$$
\begin{aligned}
\phi_{n}(t) & =\mathbb{E}\left[\exp \left(i t p \sqrt{n}\left(\prod_{k=1}^{l}\left[1-\mathbf{F}\left(\hat{q}_{k-1}, \hat{q}_{k}\right)\right]-p^{l}\right)\right) \mathbb{E}\left[\exp \left(i t p^{l} \sqrt{n}\left(1-\mathbf{F}\left(\hat{q}_{l}, \hat{q}_{l+1}\right)-p\right)\right) \mid \hat{q}_{1}, \ldots, \hat{q}_{l}\right]\right] \\
& =\mathbb{E}\left[\exp \left(i t p \sqrt{n}\left(\prod_{k=1}^{l}\left[1-\mathbf{F}\left(\hat{q}_{k-1}, \hat{q}_{k}\right)\right]-p^{l}\right)\right) \mathbb{E}\left[\exp \left(i t p^{l} \sqrt{n}\left(1-\mathbf{F}\left(\hat{q}_{l}, \hat{q}_{l+1}\right)-p\right)\right) \mid \hat{q}_{l}\right]\right]
\end{aligned}
$$

PI n ${ }^{\circ} 1747$ 
Lemma 5 ensures that we can write the last term in another way :

$$
\mathbb{E}\left[\exp \left(i t p^{l} \sqrt{n}\left(1-\mathbf{F}\left(\hat{q}_{l}, \hat{q}_{l+1}\right)-p\right)\right) \mid \hat{q}_{l}\right]=\mathbb{E}\left[\exp \left(i t p^{l} \sqrt{n}\left(1-U_{n,\left(n-k_{n}\right)}\right)\right],\right.
$$

which is a deterministic complex number. Thus

$$
\phi_{n}(t)=\mathbb{E}\left[\exp \left(i t p \sqrt{n}\left(\prod_{k=1}^{l}\left[1-\mathbf{F}\left(\hat{q}_{k-1}, \hat{q}_{k}\right)\right]-p^{l}\right)\right)\right] \mathbb{E}\left[\exp \left(i t p^{l} \sqrt{n}\left(1-U_{n,\left(n-k_{n}\right)}\right)\right] .\right.
$$

And now we just have to show that both terms have a Gaussian limit. By induction hypothesis, we know that

$$
p \sqrt{n}\left(\prod_{k=1}^{l}\left[1-\mathbf{F}\left(\hat{q}_{k-1}, \hat{q}_{k}\right)\right]-p^{l}\right) \underset{n \rightarrow+\infty}{\stackrel{\mathcal{D}}{\longrightarrow}} \mathcal{N}\left(0, p^{2} \sigma_{l}^{2}\right) .
$$

By lemma 3, we know that

$$
p^{l} \sqrt{n}\left(1-U_{n,\left(n-k_{n}\right)}\right) \underset{n \rightarrow+\infty}{\stackrel{\mathcal{D}}{\longrightarrow}} \mathcal{N}\left(0, p^{2 l+1}(1-p)\right) .
$$

Thus

$p \sqrt{n}\left(\prod_{k=1}^{l}\left[1-\mathbf{F}\left(\hat{q}_{k-1}, \hat{q}_{k}\right)\right]-p^{l}+p^{l} \sqrt{n}\left(1-\mathbf{F}\left(\hat{q}_{l}, \hat{q}_{l+1}\right)-p\right) \underset{n \rightarrow+\infty}{\stackrel{\mathcal{D}}{\longrightarrow}} \mathcal{N}\left(0, p^{2} \sigma_{l}^{2}+p^{2 l+1}(1-p)\right)\right.$.

By lemma 4, we conclude that

$$
\sqrt{n}\left(\prod_{k=1}^{l+1}\left[1-\mathbf{F}\left(\hat{q}_{k-1}, \hat{q}_{k}\right)\right]-p^{l+1}\right) \underset{n \rightarrow+\infty}{\stackrel{\mathcal{D}}{\longrightarrow}} \mathcal{N}\left(0, \sigma_{l+1}^{2}\right),
$$

with $\sigma_{l+1}^{2}=p^{2} \sigma_{l}^{2}+p^{2 l+1}(1-p)$. From this recursion, we deduce :

$$
\sigma_{N}^{2}=N p^{2 N-1}(1-p) .
$$

Now we deal with the last step. Let $N^{*}$ be the (random) number of steps of the algorithm. Let us first suppose that $\frac{\log \alpha}{\log p}$ is not an integer and consider again $N=\left\lfloor\frac{\log \alpha}{\log p}\right\rfloor$. Let us first assume that the algorithm is deterministically stopped after $(N+1)$ steps. Then the estimate is $\hat{\alpha}_{d, N}=p^{N} \hat{\alpha}_{N}$, where

$$
\hat{\alpha}_{N}=\frac{1}{n} \sum_{j=1}^{n} \mathbb{1}_{\left\{S_{n, j}^{N} \geq M\right\}} .
$$

The variables $\mathbb{1}_{\left\{S_{n, j}^{N} \geq M\right\}}$ are i.i.d. Bernoulli trials, conditionally to $\hat{q}_{N}$. The parameter of the Bernoulli is

$$
\rho_{n}=\mathbb{P}\left(\mathbb{1}_{\left\{S_{n, j}^{N} \geq M\right\}}=1 \mid \hat{q}_{N}\right)=1-\mathbf{F}\left(\hat{q}_{N}, M\right)=\frac{\alpha}{1-F\left(\hat{q}_{N}\right)} .
$$


We have already shown that

$$
\sqrt{n}\left[\prod_{k=1}^{N}\left(1-\mathbf{F}\left(\hat{q}_{k-1}, \hat{q}_{k}\right)\right)-p^{N}\right] \underset{n \rightarrow+\infty}{\stackrel{\mathcal{D}}{\longrightarrow}} \mathcal{N}\left(0, \sigma_{N}^{2}\right),
$$

Then we write, using the definition of $F$ and $\mathbf{F}$ :

$$
\prod_{k=1}^{N}\left(1-\mathbf{F}\left(\hat{q}_{k-1}, \hat{q}_{k}\right)\right)=1-F\left(\hat{q}_{N}\right)
$$

Let $\rho=\alpha p^{-N}$. Using equation 3, we get:

$$
\sqrt{n}\left(\rho_{n}-\rho\right)=\alpha \sqrt{n}\left[\frac{p^{N}-\left(1-F\left(\hat{q}_{N}\right)\right)}{p^{N}\left(1-F\left(\hat{q}_{N}\right)\right)}\right] .
$$

We know from the proof of theorem1 that

$$
\frac{\alpha}{p^{N}\left(1-F\left(\hat{q}_{N}\right)\right)} \underset{n \rightarrow+\infty}{\stackrel{a . s .}{\longrightarrow}} \frac{\alpha}{p^{N}} \frac{1}{p^{N}} .
$$

So we have that

$$
\sqrt{n}\left(\rho_{n}-\rho\right) \underset{n \rightarrow+\infty}{\stackrel{\mathcal{D}}{\longrightarrow}} \mathcal{N}\left(0, \sigma_{\rho}^{2}\right)
$$

with

$$
\sigma_{\rho}^{2}=\frac{\alpha^{2}}{p^{2 N+1}} N(1-p) .
$$

Then we apply lemma 6 to get that

$$
\sqrt{n}\left(\hat{\alpha}_{d, N}-\alpha\right) \underset{n \rightarrow+\infty}{\stackrel{\mathcal{D}}{\longrightarrow}} \mathcal{N}\left(0, \sigma^{2}\right),
$$

with

$$
\sigma^{2}=\alpha^{2}\left(N \frac{1-p}{p}+\frac{1-\rho}{\rho}\right) .
$$

Then we come back to the true (random) $N^{*}$. Let us consider

$$
\tilde{\alpha}_{N}=\hat{\alpha} \mathbb{1}_{\left\{N^{*}=N\right\}}+\gamma \mathbb{1}_{\left\{N^{*} \neq N\right\}},
$$

where $\gamma$ is a random variable whose law is the law of $\hat{\alpha}_{d, N}$, conditionally to $N^{*}$ and $\hat{q}_{N^{*} \wedge N}$. Then it is quite obvious to see that $\tilde{\alpha}_{N}$ has the same law as $\hat{\alpha}_{d, N}$, implying they have both the same asymptotic behavior in distribution. On the other hand we get for all $\varepsilon>0$,

$$
\mathbb{P}\left(\left|\sqrt{n}\left(\hat{\alpha}-\tilde{\alpha}_{N}\right)\right|>\varepsilon\right) \leq \mathbb{P}\left(\left|\hat{\alpha}-\tilde{\alpha}_{N}\right|>0\right)=\mathbb{P}\left(N^{*} \neq N\right) \underset{n \rightarrow+\infty}{\longrightarrow} 0,
$$

PI ${ }^{\circ} 1747$ 
as we saw in the proof of theorem 1 that $N^{*} \underset{n \rightarrow+\infty}{\stackrel{a . s .}{\longrightarrow}} N$. Now from all this we conclude that $\sqrt{n}(\hat{\alpha}-\alpha)$ and $\sqrt{n}\left(\tilde{\alpha}_{N}-\alpha\right)$ both converge to the same limit in distribution.

If $N=\frac{\log \alpha}{\log p}$ is an integer, we combine the reasoning at the end of the proof of theorem 1 (i.e. distinction of two cases) and, in each case, the calculus of variance above to obtain the asymptotic normality with variance :

$$
\sigma^{2}=\alpha^{2} N \frac{1-p}{p}
$$

\section{Numerical example}

We have implemented this algorithm with trajectories following a Brownian process with drift. Noting $B_{t}$ a Brownian motion, the process studied is $X_{t}=B_{t}+\mu t$, with $\mu<0$ (see Figure 4). The drift was taken to have a motion going quickly to 0 . This process clearly satisfies assumption $\mathcal{H}$. Moreover, it is simple enough so that analytical results are well-known about it.

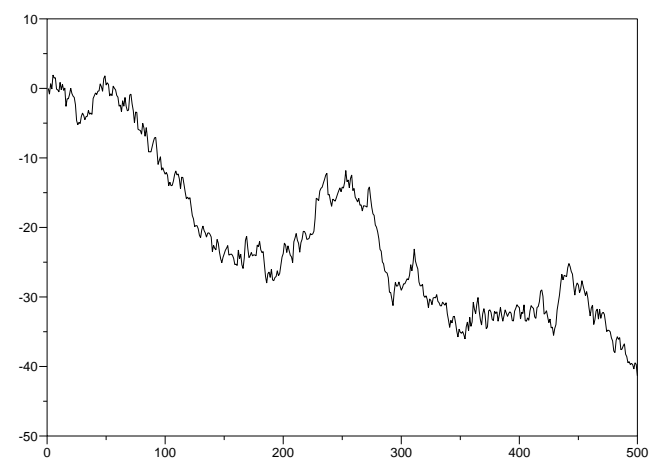

Figure 4: Brownian motion with negative drift starting from 0.

We note $H_{a, b}=H=\min \left\{s>0: X_{s} \notin[a, b]\right\}$. The expression of the probability of reaching $b$ before $a$ starting from $x \in[a, b]$ is given by [3]:

$$
\mathbb{P}_{x}\left(X_{H}=b\right)=e^{\mu(b-x)} \frac{\sinh ((x-a)|\mu|)}{\sinh ((b-a)|\mu|)}
$$


Let us compare this to our numerical results.

The first problem we had to solve was that we were considering a continuous process, which is impossible in computing. We had to choose a step $\delta t$ and consider the process at every $k \times \delta t$. This step has to be small enough to avoid clipping the process, which could introduce a bias in the estimation.

We will illustrate first the a.s. convergence. We ran our algorithm on the above example with parameters $a=0, b=12, \mu=-1, x_{0}=1$, such that the rare event probability is $\alpha \approx 2.412 \times 10^{-10}$. Figure 5 gives the relative error as a function of $n$ the number of particles. For $n=20,000$ we have an error as low as $5 \%$.

Then we illustrate the asymptotic normality. As we need to run the algorithm many times to estimate the law of the estimator, we chose a setting where $\alpha$ is not very small, but about 0.1244. Estimating of the same probability $\alpha 1000$ times gives the histogram of Figure 6 . This confirms the fact that the distribution of the estimating values tends asymptotically towards a Gaussian distribution. Then we show the convergence of the variance multiplied by $n$ on Figure 7. In this setting, we computed the asymptotic value which is about 0.0373 , as shown by the dashed line on the figure. Here we made 250 runs for each value of $n$.

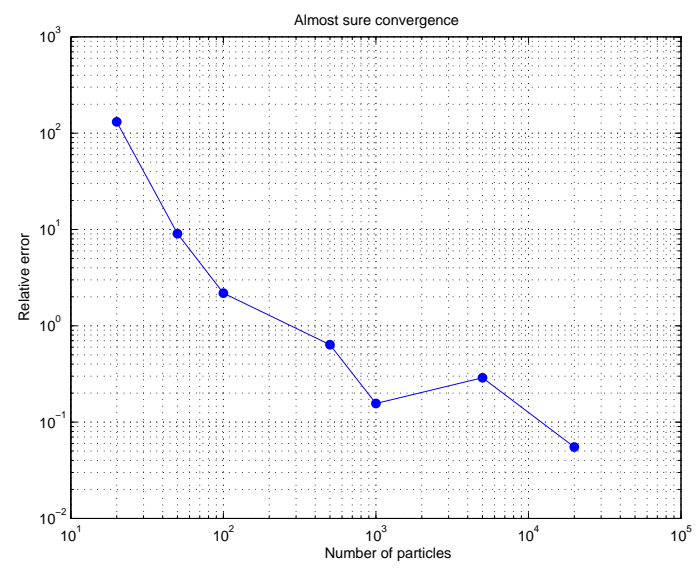

Figure 5: Almost sure convergence of the estimator as a function of $n$. 


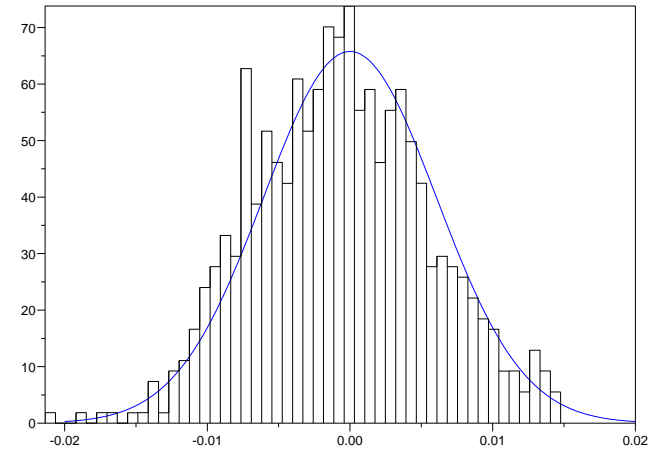

Figure 6: Histogram of the differences between $\gamma$ and its estimations, with $n=1000$, for 1000 instances. The curve represents the limit Gaussian distribution.

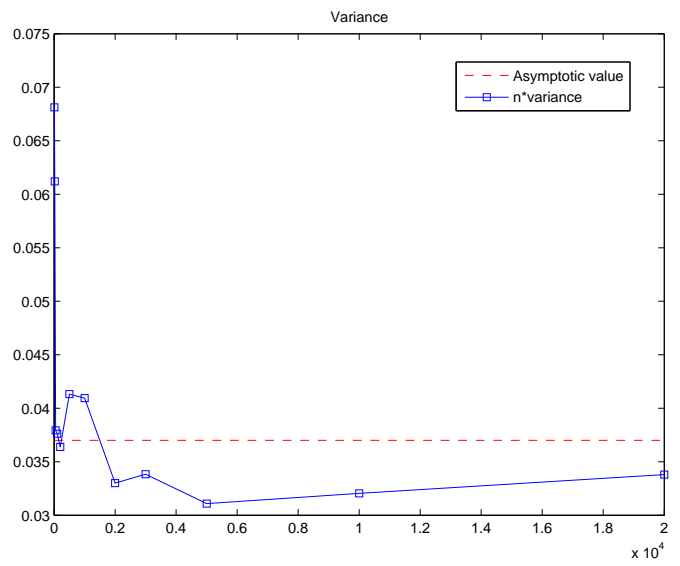

Figure 7: $n \times$ variance of the estimator as a function of $n$. 


\section{Comparison with existing algorithms}

\subsection{Complexity and variance}

Now we want to have an idea about the complexity of the algorithm. To this aim let us consider the following simplification : we suppose that the simulation of a trajectory $\left(X_{t}^{x}\right)_{0 \leq t \leq T_{0}}$, between its starting point $x>0$ and the first time $T_{0}$ it hits 0 , has a cost $C$ which is approximately constant, independent of the initial condition $x$.

For the first step, with the assumption above, the cost for simulating $n$ trajectories is linear in $n$. The finding of the maxima of the $n$ trajectories is also linear in $n$. The sorting of these maxima is, in expectation, in $O(n \log n)$. Thus the first step of the algorithm has an expected complexity in $O(n \log n)$. For $n$ large enough, there is a finite number of steps, that is $(N+1)$, with $N=\left\lfloor\frac{\log \alpha}{\log p}\right\rfloor$. Finally the total cost of the algorithm is in $O(n \log n)$ operations.

We are now interested in the precision of the estimator. We have seen above that the variance of $\hat{\alpha}$ is

$$
\sigma^{2}=\alpha^{2}\left(N \frac{1-p}{p}+\frac{1-\rho}{\rho}\right),
$$

with $p<\rho<1$. If for simplicity we suppose that $\alpha=p^{N}$, then the normalized variance is

$$
\frac{\sigma^{2}}{\alpha^{2}}=N \frac{1-p}{p} \approx \frac{\log \alpha}{\log p} \cdot \frac{1-p}{p} .
$$

In real life applications, the only parameter that is fixed a priori is the small probability $\alpha$ to estimate. So the question is : what is the optimal choice for $p$ ? A straightforward study of the variance for $p$ varying between $\alpha$ and 1 proves that $\sigma^{2}$ decreases when $p$ goes to one.

This result is intuitively clear and merely says that if we want a precise estimate for $\alpha$, we just have to put a lot of intermediate levels. But, of course, the complexity of the algorithm is then increasing since the number of levels is $\frac{\log \alpha}{\log p}$. So the choice of the parameter $p$, or equivalently the choice of the number $N$ of levels, depends on what we want : a precise estimate or a quick algorithm.

\subsection{Classical multilevel splitting}

A usual way for estimating rare event probability is the multilevel splitting algorithm. The splitting idea is widespread in Monte Carlo methods, see for instance [11], p.131. Its application to rare event estimation is first due to Kahn and Harris in the setting of particle transmission [12]. In 1970, Bayes proposed to apply it in the field of waiting queues [2] $]^{1}$. This idea was rediscovered twenty years later by Villén-Altamirano and Villén-Altamirano

\footnotetext{
${ }^{1}$ Unfortunately, he introduces some confusion in his paper by using the term "importance sampling" for what is generally named "importance splitting".
} 
[17] : this is what they call the RESTART method (REpetitive Simulation Trials After Reaching Thresholds) with applications in telecommunication traffic and reliability. In a simplified context, the idea has been theoretically studied by Glasserman et al. in several papers [8][9][10], and more recently by Lagnoux [13]. We refer the reader to [10] for a precise discussion and many references about splitting algorithms and their applications.

We can describe the algorithm in the simplified form of these last authors on our Markov process example. Let us denote $A$ the event "reaching $M$ before 0 , starting from $x_{0}$ ", then consider $A=A_{N} \subset A_{N-1} \subset \cdots \subset A_{1}$ an increasing sequence of events. For us, this is equivalent to considering a decreasing sequence of levels $M=M_{N}>M_{N-1}>$ $\cdots>M_{1}>x_{0}$, each $M_{i}$ being the threshold between $A_{i-1}$ and $A_{i}$. Let $p_{1}=\mathbb{P}\left(A_{1}\right)$ and $p_{i+1}=\mathbb{P}\left(A_{i+1} \mid A_{i}\right)$. These probabilities are bigger than $\alpha=\mathbb{P}(A)$ and thus easier to estimate. Moreover, since the process is Markov, the following product decomposition holds :

$$
\alpha=p_{1} p_{2} \ldots p_{N} \text {. }
$$

In the version of Lagnoux, the idea of splitting is to simulate $n$ paths starting from $x_{0}$, to duplicate $R_{1}$ times those who have reached $M_{1}$ before 0 (which happens with probability $p_{1}$ ), then to duplicate $R_{2}$ times those who have reached $M_{2}$ before 0 starting from $M_{1}$ (which happens with probability $p_{2}$ ), etc. (see Figure 8 ).
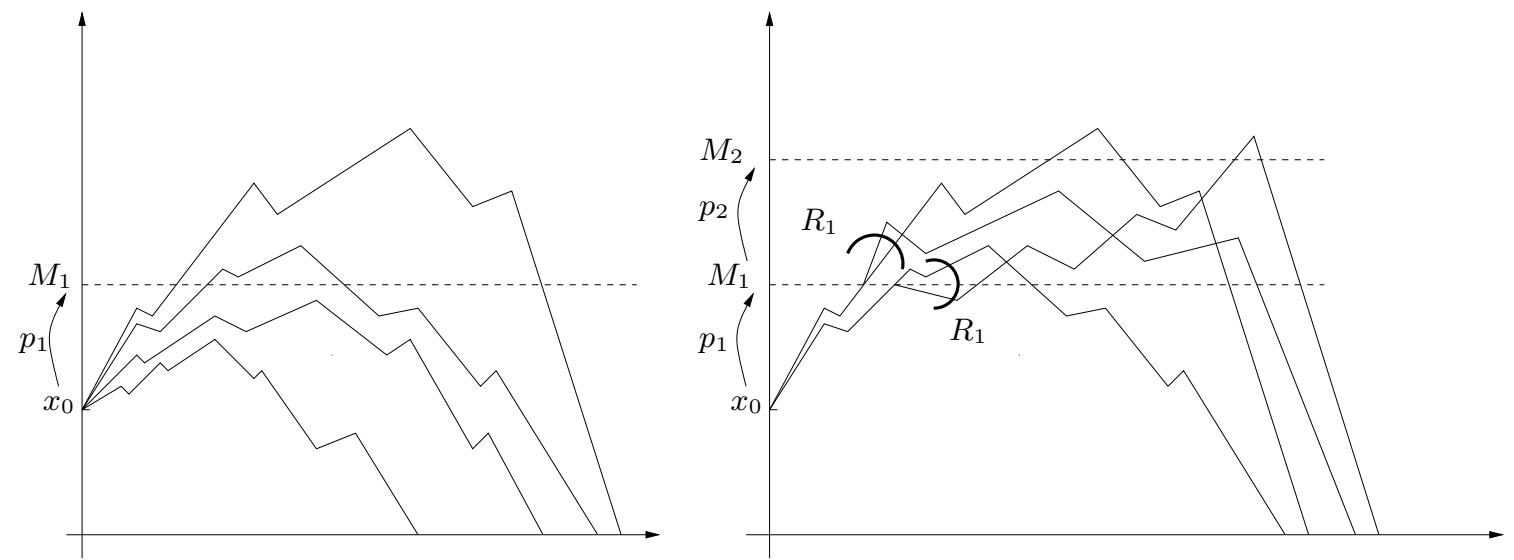

Figure 8: Multilevel splitting as seen by Lagnoux, with $n=4$ and $R_{1}=2$.

An unbiased estimator of $\alpha$ is

$$
\tilde{\alpha}=\frac{n_{A}}{n R_{1} \ldots R_{N}},
$$

where $n_{A}$ is the number of trajectories that have reached $M$ before 0 . The complexity of the algorithm is this time in $O(n)$, which is less than for the adaptive multilevel splitting 
algorithm.

As Glasserman et al. noticed, one can also see the multilevel splitting method in terms of branching processes. Suppose there is initially one ancestor, his offspring has a binomial distribution $\mathcal{B}\left(R_{1}, p_{1}\right)$, each child has himself an offspring with binomial distribution $\mathcal{B}\left(R_{2}, p_{2}\right)$, etc. (see Figure 9). Compare to the version of Lagnoux, we see that it is the same idea, there is only a shift of one step in the splitting.

Suppose that if the number $N$ and the levels $\left(M_{1}, \ldots, M_{N}\right)$ are fixed, we can compute the probabilities $\left(p_{1}, \ldots, p_{N}\right)$, and vice versa. Then, if the sequence $\left(R_{1}, \ldots, R_{N}\right)$ is fixed also, we can compute the variance of the estimator $\tilde{\alpha}$ : either through direct calculus [13], or via the theory of branching processes [10]. The natural question that arises then is : what is the best choice for these sequences, in terms of estimator variance and complexity? Glasserman et al. have shown that asymptotically when $\alpha \rightarrow 0$, the best thing we have to do is to take all the $p_{i}$ 's equal to $p=\alpha^{1 / N}$ and all the $R_{i}$ 's equal to $R=1 / p$ (if this quantity is not an integer, just randomize the algorithm so that $\mathbb{E}[R]=1 / p)$. Lagnoux has shown the same result without any asymptotics on $\alpha$. In terms of branching processes, this result says that the best compromise between complexity and variance is reached in the critical regime. No surprise in this story : if $R>1 / p$, the variance is smaller than simple Monte Carlo, but the number of paths will explode $\mathrm{e}^{2}$, and if $R<1 / p$, there is no substantial gain in estimation compared to naive Monte Carlo.
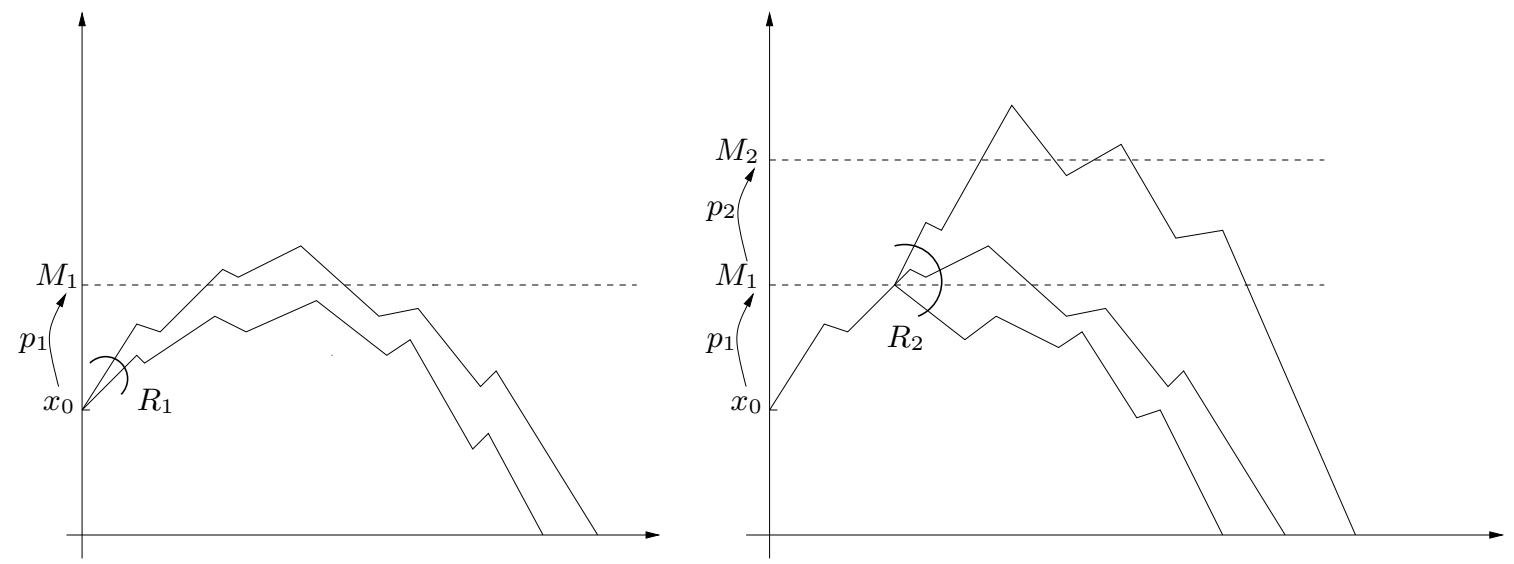

Figure 9: Multilevel splitting as seen by Glasserman, with $R_{1}=2$ and $R_{2}=3$.

\footnotetext{
${ }^{2}$ in this case, most of the computing time is spent with highly correlated trajectories
} 
In relation with this last point, we could have described our algorithm in a slightly more general way, that means : with parameters $\left(k_{1}, n_{1}\right), \ldots,\left(k_{N}, n_{N}\right)$ instead of the same couple $(k, n)$ at each step. Anyway, when $n$ and $k$ go to infinity so that the ratio $k / n$ goes to $p$, it is clear that we get closer and closer to the classical splitting algorithm with regular levels. Thus, the result obtained by preceding authors on the classical splitting algorithm shows that the best thing we have to do in our case is to keep the same couple $(k, n)$ at each step.

For the sake of simplicity, let us suppose again that $\alpha=p^{N}$, with $R=\frac{1}{p}$ an integer. Then the variance $s^{2}$ of the estimator $\tilde{\alpha}$ in classical multilevel splitting is just the same ${ }^{3}$ as for our $\hat{\alpha}$ :

$$
\frac{s^{2}}{\alpha^{2}}=N \frac{1-p}{p}
$$

The difference between the variances of $\hat{\alpha}$ and $\tilde{\alpha}$ is the following : since the adaptive version needs interactions between the $n$ trajectories, its variance is asymptotic when $n$ goes to infinity. This is not the case with classical splitting, where the trajectories are independent. On the other hand, the above formula is only a best case for classical multilevel splitting (regular levels), whereas it is always asymptotically granted in the adaptive version, as long as $k / n$ is kept fixed. In other words, in terms of estimators fluctuations, classical multilevel splitting will never perform better than adaptive multilevel splitting.

Indeed, in real life applications, it is unfortunately impossible to have regular levels: the systems are usually so complex that any analytic calculus about them is just out of question. In this context, our adaptive algorithm is very useful : the levels $\left(\hat{q}_{1}, \ldots, \hat{q}_{N}\right)$ are determined during the algorithm and they are in fact approximations of the true quantiles $\left(M_{1}, \ldots, M_{N}\right)$. So, at the expense of a multiplying factor $\log n$ in the complexity, we have an algorithm that is really suitable for applications.

Concerning the issue of fixing the levels, the authors discovered a posteriori the thesis of Garvels [6]. In section 3.3.1., he proposes to estimate them during a trial run, in the same way as we do, and then to apply classical splitting. He writes: "Care has to be taken that enough samples are used determining the thresholds, otherwise the thresholds may become biased and force the real simulation in the wrong area. A good rule of thumb is to devote $10 \%$ of the simulation effort to a trial run which will determine all the thresholds." To our knowledge, he does not mention that both tasks can be treated simultaneously, with a consistency result and an "optimal" asymptotic variance.

\subsection{Multilevel splitting as a Feynmac-Kac model}

Another variant of multilevel splitting has been recently proposed by Del Moral [4], [5]. Like in classical multilevel splitting, it requires to fix the thresholds before beginning simu-

\footnotetext{
${ }^{3}$ Note that the formula p.589 in [10] seems to be different, but this is only due to the first step of their algorithm.
} 

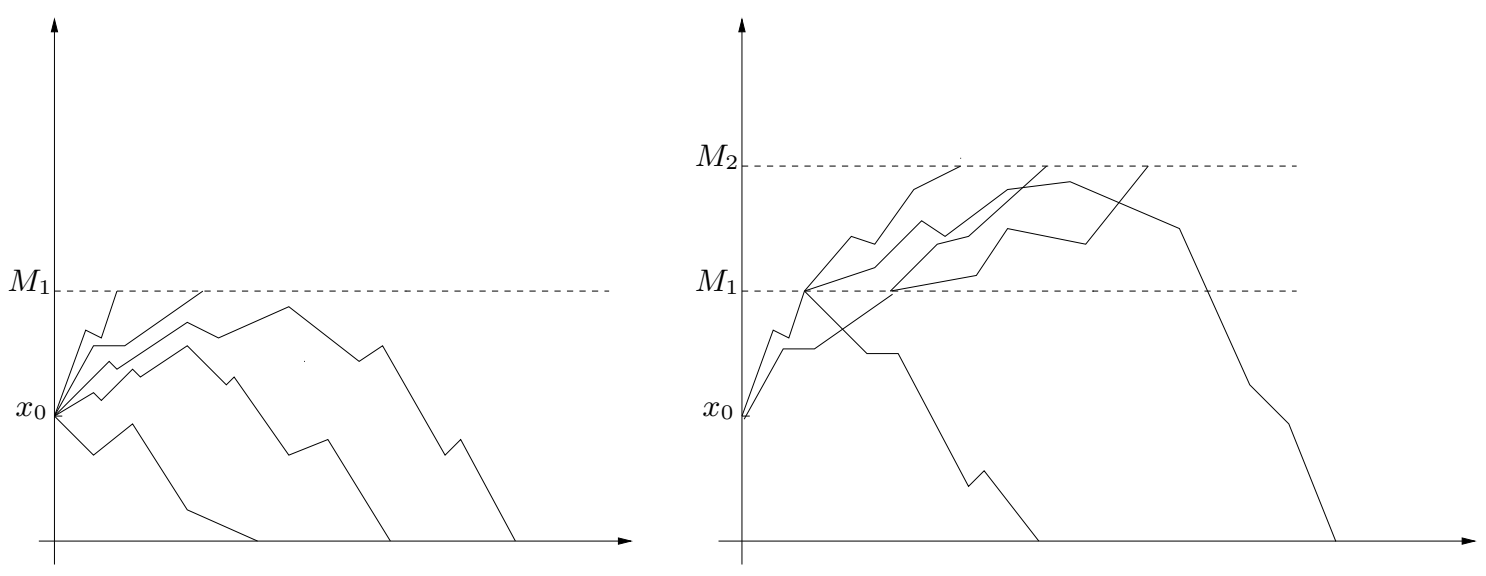

Figure 10: Multilevel splitting as a Feynmac-Kac model, with $n=5$.

lations (in other words, it is not adaptive). Anyway, one of the benefits of this version is to connect multilevel splitting techniques with interacting particle systems approximations of Feynman-Kac distributions. This last topic has been intensively studied since the beginning of the 90's, see [4], so that precise and general results can be transposed in the field of rare event analysis.

We can briefly describe the algorithm in the context of the above mentioned Markov process: suppose the number $N$ and the levels $M_{1}<\cdots<M_{N}=M$ are fixed, like in classical splitting. At time 0 , the algorithm starts with $n$ independent copies of the process $X$ that are stopped when they reach 0 or $M_{1}$, whatever occurs the first. The particles which reach 0 before $M_{1}$ are killed and and randomly redistributed among those having reached the first level, producing offsprings. If the whole system is killed, the algorithm is stopped. Otherwise, with the offprings, there are still $n$ particles at the first level $M_{1}$. In a second step, the $n$ particles in the first level evolve according to the same rule of the process $X$. Here again particles which reach 0 before $M_{2}$ are killed and for each killed one we randomly choose one of the particles that have reached the second level and add an offspring to it (see Figure 10). Then the process goes on to the next level, and so on until the rare event is reached.

For comparison, the cost is the same as for classical splitting, in $O(n)$. The asymptotic normality of the estimator is proved, with the same variance as before, i.e. $N \cdot \frac{1-p}{p}$ (by adapting for instance theorem 12.2.2 in [4] in our framework). 


\subsection{Generalization}

The assumptions made to prove the consistency and the asymptotic normality of $\hat{\alpha}$ are mainly : the process is Markov, the distribution function $F$ of its supremum is continuous, the process is in one dimension.

Even if, for now, we do not have theoretical results in more general cases, the algorithm itself is quite versatile. In the following, we briefly present two examples where the above assumptions are not satisfied, but where first experimentations give promising results.
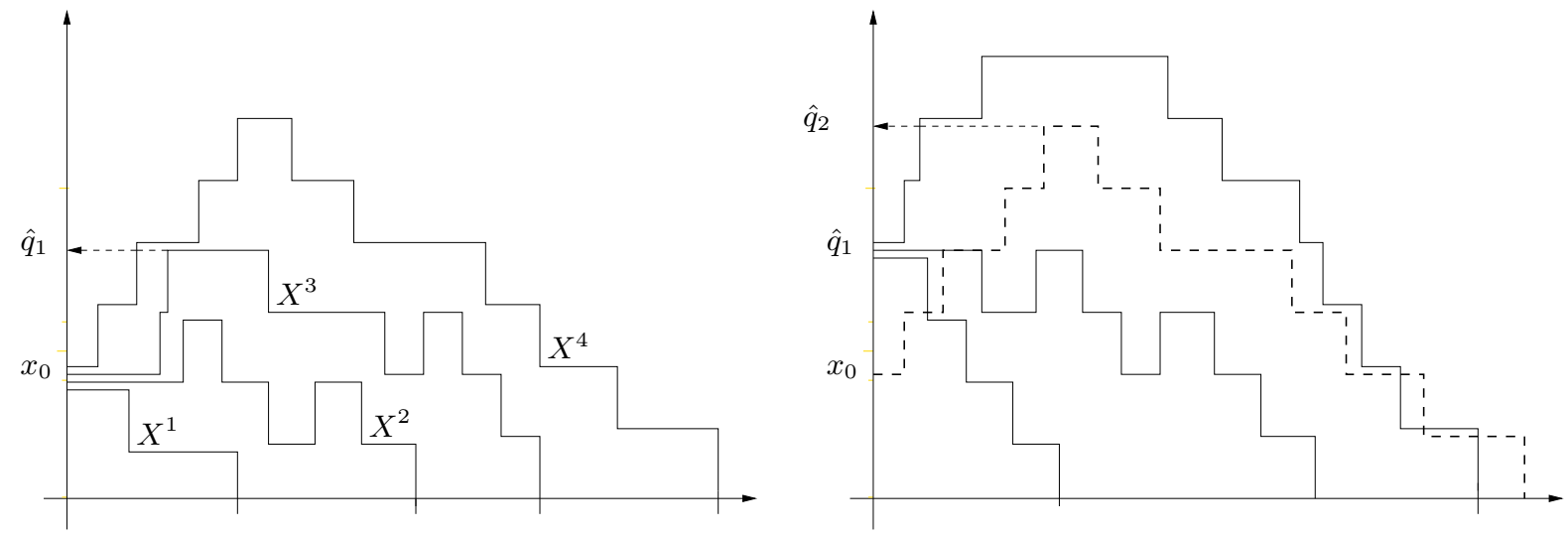

Figure 11: Adaptive algorithm for a waiting queue, with $n=4$ and $k=1$.

\section{Examples.}

\section{- Saturation of a waiting queue buffer}

Consider a queue with 0 as a positive recurrent state. In other words, the tasks are treated faster than the clients arrive, so that the system is stable. Suppose moreover that the system has a huge but finite capacity, so that the saturation will be a rare event. We want to estimate its probability with adaptive splitting.

The process $\left(X_{t}\right)_{t \geq 0}$ is the number of clients in the system. Note first that in general this process is not Markov. Secondly, $\left(X_{t}\right)_{t \geq 0}$ is not continuous. Consequently, we cannot apply directly our theoretical results. Nevertheless, there is no problem in adapting our algorithm in this context. The first steps are described in Figure 11.

\section{- Self avoiding walks}

Let us now mention a two-dimensional problem. We consider self-avoiding walks in $\mathbb{Z}^{2}$, which serves as a model for molecular chains. We want to calculate the probability 
of having long chains and draw some of them.

Here again, even if theory is only established for one-dimensional problems, adapting the algorithm is straightforward. The algorithm is described in Figure 12. The supremum of the trajectory in previous examples is just replaced by the length of the random walk until it hits himself. Note that in general, for multidimensional problem$\mathrm{s}$, this is the difficult question : which criterion does really measure the fact that we are approaching the rare event ? That is what Garvels et al. call "the choice of the Importance Function" [7].
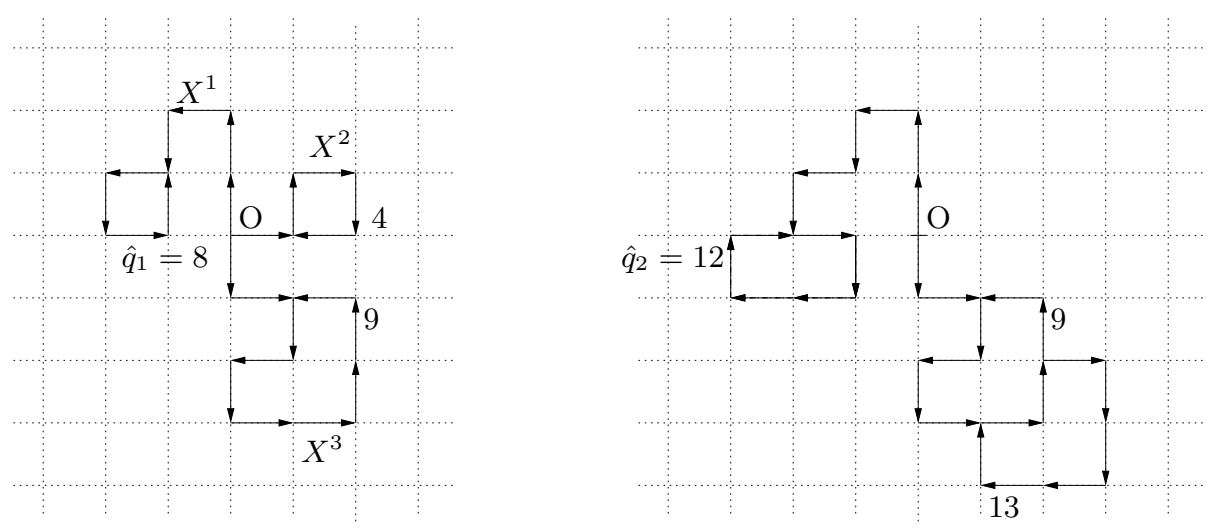

Figure 12: Adaptive algorithm for self-avoiding walks, with $n=3$ and $k=1$.

\section{Conclusion}

We have presented a new algorithm for rare event analysis. This algorithm belongs to the multilevel family, but does not require the splitting levels to be set in advance. Instead it adapts them on the fly during the simulation. In the one dimensional case, we showed that there is no loss in variance compared to the other algorithms with optimal splitting levels, and only a slight increase in complexity.

\section{Acknowledgments}

The authors would like to thank Philippe Berthet for valuable discussions about empirical processes, and Hélène Topart for her participation to the numerical simulations. 


\section{References}

[1] B.C. Arnold, N. Balakrishnan, and H.N. Nagaraja. A first course in order statistics. Wiley Series in Probability and Mathematical Statistics: Probability and Mathematical Statistics. John Wiley \& Sons Inc., New York, 1992.

[2] A. J. Bayes. Statistical techniques for simulation models. Australian Comput. J., 2:180$184,1970$.

[3] A. N. Borodin and P. Salminen. Handbook of Brownian Motion - Facts end Formulae. Birkhäuser Verlag Basel Boston Berlin, 1996.

[4] P. Del Moral. Feynman-Kac formulae, Genealogical and interacting particle systems with applications. Probability and its Applications. Springer-Verlag, New York, 2004.

[5] P. Del Moral and J. Garnier. Genealogical particle analysis of rare events. submitted to the Annals of Applied Probability, 2004. http://www.lsp.ups-tlse.fr/Garnier/.

[6] M.J.J. Garvels. The splitting method in rare event simulation. Thesis, University of Twente, Twente, May 2000. http://www.math.utwente.nl/dos/sor/AiO's/Garvels/Thesis.

[7] M.J.J. Garvels, D.P. Kroese, and J-K.C.W. Van Ommeren. On the importance function in splitting simulation. European Transactions on Telecommunications, 13(4):363-371, 2002. http://www.maths.uq.edu.au/ kroese/.

[8] P. Glasserman, P. Heidelberger, P. Shahabuddin, and T. Zajic. A large deviations perspective on the efficiency of multilevel splitting. IEEE Trans. Automat. Control, 43(12):1666-1679, 1998.

[9] P. Glasserman, P. Heidelberger, P. Shahabuddin, and T. Zajic. A look at multilevel splitting. In Monte Carlo and quasi-Monte Carlo methods 1996 (Salzburg), volume 127 of Lecture Notes in Statist., pages 98-108. Springer, New York, 1998.

[10] P. Glasserman, P. Heidelberger, P. Shahabuddin, and T. Zajic. Multilevel splitting for estimating rare event probabilities. Oper. Res., 47(4):585-600, 1999.

[11] J. M. Hammersley and D. C. Handscomb. Monte Carlo methods. Methuen \& Co. Ltd., London, 1965.

[12] H. Kahn and T.E. Harris. Estimation of particle transmission by random sampling. National Bureau of Standards Appl. Math. Series, 12:27-30, 1951.

[13] A. Lagnoux. Rare event simulation. PEIS, to appear, 2005. http://www.lsp.upstlse.fr/Fp/Lagnoux/.

[14] P. Massart. The tight constant in the Dvoretzky-Kiefer-Wolfowitz inequality. Ann. Probab., 18(3):1269-1283, 1990. 
[15] G.R. Shorack and J.A. Wellner. Empirical processes with applications to statistics. Wiley Series in Probability and Mathematical Statistics: Probability and Mathematical Statistics. John Wiley \& Sons Inc., New York, 1986.

[16] A.W. van der Vaart. Asymptotic statistics. Cambridge Series in Statistical and Probabilistic Mathematics. Cambridge University Press, Cambridge, 1998.

[17] M. Villén-Altamirano and J. Villén-Altamirano. RESTART : a straightforward method for fast simulation of rare events. In Jeffrey D. Tew, Mani S. Manivannan, Deborah A. Sadowski, and Andrew F. Seila, editors, Proceedings of the 1994 Winter Simulation Conference, Orlando 1994, pages 282-289, December 1994. 